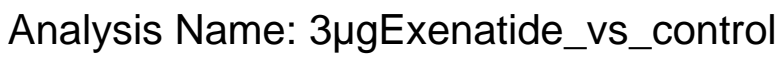

Analysis Creation Date: 2013-12-06

Build version: 261899

Content version: 17199142 (Release Date: 2013-09-17)

\title{
Analysis settings
}

View

Reference set: Mouse Genome 430 2.0 Array

Relationship to include: Direct and Indirect

Includes Endogenous Chemicals

Optional Analyses: My Pathways My List

Filter Summary:

Consider only relationships where

(confidence $=$ Experimentally Observed) AND

(data sources = An Open Access Database of Genome-wide Association Results OR BIND OR BIOGRID OR Catalogue Of Somatic Mutations In Cancer (COSMIC) OR Chemical Carcinogenesis Research Information System (CCRIS) OR ClinicalTrials.gov OR ClinVar OR Cognia OR DIP OR DrugBank OR Gene Ontology (GO) OR GVK Biosciences OR Hazardous Substances Data Bank (HSDB) OR HumanCyc OR Ingenuity Expert Findings OR Ingenuity ExpertAssist Findings OR INTACT OR Interactome studies OR MINT OR MIPS OR miRBase OR miRecords OR Mouse Genome Database (MGD) OR Obesity Gene Map Database OR Online Mendelian Inheritance in Man (OMIM) OR TarBase OR TargetScan Human)

Cutoff:

Fold Change $=1.300$

$\mathrm{p}$-value $=5.00 \mathrm{E}-02$ 


\section{Top Networks}

ID Associated Network Functions

Energy Production, Lipid Metabolism, Small Molecule Biochemistry

Behavior, Nervous System Development and Function, DNA Replication, Recombination, and Repair

Organ Morphology, Tissue Development, Gastrointestinal Disease

Cancer, Hematological Disease, Reproductive System Disease

40

$5 \quad$ Lipid Metabolism, Small Molecule Biochemistry, Connective Tissue Disorders 
Top Diseases and Bio Functions

\section{Diseases and Disorders}

Name

p-value

\#

Molecule

1.42E-05 - 1.69E-02 12

Connective Tissue Disorders

1.42E-05 - 1.95E-02 67

Metabolic Disease

2.72E-05 - 2.81E-02 193

Gastrointestinal Disease

$2.72 \mathrm{E}-05-2.80 \mathrm{E}-0220$

Neurological Disease

$6.42 \mathrm{E}-05-1.29 \mathrm{E}-0278$

\section{Molecular and Cellular Functions}

Name

p-value

\# Molecule

Cell Death and Survival

7.77E-05 - 2.85E-02 186

Cell Morphology

Cell-To-Cell Signaling and Interaction

7.51E-04 - 2.58E-02 96

Lipid Metabolism

7.51E-04 - 2.69E-02 32

Nucleic Acid Metabolism

9.54E-04 - 2.69E-02 67

9.54E-04 - 2.58E-02 14

\section{Physiological System Development and Function}

Name

p-value

\#

Molecule

Connective Tissue Development and Function

4.00E-04 - 2.79E-02 33

Tissue Development

4.00E-04 - 2.58E-02 62

Organismal Survival

4.08E-04 - 2.50E-02 143

Digestive System Development and Function

5.84E-04 - 2.80E-02 60

(c) 2000-2014 Ingenuity Systems, Inc. All rights reserved. 


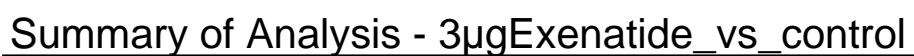

\section{Top Canonical Pathways}

Name

p-value

Ratio

Fatty Acid $\beta$-oxidation I

7.44E-05

$7 / 45$

Caveolar-mediated Endocytosis Signaling

2.01E-04

$(0.156)$

Virus Entry via Endocytic Pathways

3.11E-04

RAR Activation

3.58E-03

Phenylethylamine Degradation I

4.08E-03

\section{Top Molecules}

\section{Fold Change up-regulated}

\begin{tabular}{ll} 
Molecules & Exp. Value \\
\hline ZBTB16 & Chart \\
Acot1 & \\
UPP2 & \\
NR1D2 & \\
FAM107A & \\
ECH1 &
\end{tabular}


Summary of Analysis - $3 \mu \mathrm{gExenatide} \mathrm{vs} \mathrm{control}$

RGN

SPHK1

$\uparrow 2.229$

BMP4

$\uparrow 2.212$

CMBL

$\uparrow 2.184$

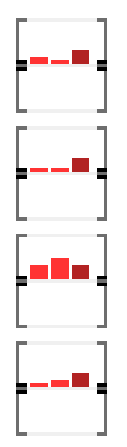

\section{Fold Change down-regulated}

\begin{tabular}{|c|c|c|}
\hline Molecules & Exp. Value & $\begin{array}{l}\text { Exp. } \\
\text { Chart }\end{array}$ \\
\hline GADD45G & $\downarrow-3.611$ & \\
\hline ELOVL6 & $\downarrow-3.231$ & \\
\hline HSPA8 & $\downarrow-3.108$ & \\
\hline SOAT1 & $\downarrow-2.811$ & \\
\hline CADM3 & $\downarrow-2.573$ & \\
\hline CRY1 & $\downarrow-2.535$ & \\
\hline MT1E & $\downarrow-2.431$ & \\
\hline ILDR2 & $\downarrow-2.392$ & \\
\hline SGK1 & $\downarrow-2.384$ & \\
\hline P2RY6 & $\downarrow-2.360$ & \\
\hline
\end{tabular}


Top Upstream Regulators

Upstream Regulator

ACOX1

EHHADH

beta-estradiol

ciprofibrate

POR p-value of overlap Predicted

Activation State

3.44E-06

8.70E-06

1.13E-05

1.38E-05

1.47E-05 


\section{Top My Lists}

Name

p-value

Ratio

My List angiogenesis 769

Top My Pathways

Name

p-value

Ratio

\section{Top Tox Lists}

Name

Fatty Acid Metabolism

\begin{tabular}{cl} 
p-value & Ratio \\
\hline 2.81E-05 & $14 / 118$ \\
& $(0.119)$ \\
3.02E-03 & $6 / 42$ \\
& $(0.143)$ \\
3.99E-03 & $14 / 176$ \\
& $(0.08)$ \\
4.63E-02 & $7 / 95$ \\
& $(0.074)$ \\
5E-02 & $15 / 262$ \\
& $(0.057)$
\end{tabular}

Pro-Apoptosis

RAR Activation

p53 Signaling

Liver Necrosis/Cell Death

$(0.057)$ 


\section{Top Tox Functions}

\section{Assays: Clinical Chemistry and Hematology}

Name

p-value

\#

Molecule

Increased Levels of Red Blood Cells

6.86E-03 - 6.86E-03 9

Decreased Levels of Albumin

3.74E-02 - 2.90E-01 2

Increased Levels of AST

3.67E-01 - 3.67E-01 1

Increased Levels of Blood Urea Nitrogen

4.13E-01 - 4.13E-01 1

Increased Levels of Potassium

4.35E-01 - 4.35E-01 1

\section{Cardiotoxicity}

Name

p-value

olecule

Cardiac Inflammation

2.39E-02 - 4.57E-01 5

Cardiac Degeneration

3.36E-02 - 3.36E-02 2

Cardiac Arrythmia

3.74E-02 - 1.00E00 5

Cardiac Fibrosis

3.74E-02 - 5.84E-01 9

Cardiac Proliferation

3.74E-02 - 4.60E-01 4

\section{Hepatotoxicity}

Name

p-value

\#

Molecule

Liver Steatosis

4.87E-04 - 1.24E-01 21

Liver Regeneration

5.84E-04 - 1.08E-01 9

Liver Inflammation/Hepatitis

$1.68 \mathrm{E}-03-5.17 \mathrm{E}-0113$

Hepatocellular Carcinoma

1.16E-02 - 3.67E-01 25

(c) 2000-2014 Ingenuity Systems, Inc. All rights reserved. 


\section{Nephrotoxicity}

Name

p-value

\#

Molecule

Renal Necrosis/Cell Death

5.12E-03 - 1.00E00 19

Glomerular Injury

Nephrosis

3.74E-02 - 5.40E-01 12

Renal Enlargement

$3.74 \mathrm{E}-02-3.39 \mathrm{E}-013$

Renal Inflammation

3.74E-02 - 1.08E-01 1

$3.74 \mathrm{E}-02-1.00 \mathrm{E} 008$ 